\title{
Our experience in ocular biometry in congenital and infantile cataract
}

\author{
Cristina Nicula*, Nicula Dorin, Pop Radu Nicolae and Decsei-Nagy Andreea \\ Cluj-Napoca University Ophthalmology Clinic, Cluj-Napoca, Romania
}

\begin{abstract}
Purpose: To establish the importance of ocular biometry in congenital and infantile cataract upon the visual outcomes.

Methods: The study was a retrospective study upon patients with congenital and infantile cataract. The study group included 58 eyes from 36 children. For cooperative children, for the ocular biometry we used the OCUSCAN biometer (Alcon, Forth Worth Tx). In very young children we used B-scan ultrasonography to measure the axial length (AXL) of the eye. The target refraction was hyperopia, concording with the age.

Results: The measurement of axial length revealed an eye between 20 and above $25 \mathrm{~mm}$. The correlation between axial length and age showed a rapid increase in the first 12 months, a smaller increase between 100-150 months and stabilization after 200 months (statistically significant-medium correlation). The correlation between keratometry and age showed higher values of keratometry at younger children, with a significant decrease in the first 12 months and a smaller rate of decrease after 24 months of age (statistically significant-high correlation).
\end{abstract}

Conclusion: our study shows that the axial length increases during childhood, especially in the first 2 years and the corneal curvature decreases from birth to different ages, parameters which induce the target refraction.

\section{Introduction}

Congenital cataract is defined as the lens opacification which appears at birth or in the first months of life [1]. Infantile cataract represents the opacification of the lens which appears after 2 years of age.

Congenital and infantile cataracts produce deprivation amblyopia and can thus cause long life visual impairment [2]. Visual deprivation has a significant impact on the development of fixation stability. That's why it's necessary an early diagnosis and referral for surgery in order to assure an accurate optical rehabilitation.

Appropriate refractive outcome following pediatric cataract surgery with intraocular lens implantation (IOL) requires accurate biometry [3], which should be carried-out by personnel experienced in biometry in infants and children. The calculation of IOL power in infants below 1 years of age is prone to error [4]. Main reasons for sub-optical errors were: technical difficulties in measuring biometric parameters, the use of formulas and incomplete knowledge of the development of the eye in the first years of life. That's why managing cataracts in children remains a challenge. The timing of the surgical treatment is crucial for the visual development and successful rehabilitation of children.

\section{Material and method}

The study was a retrospective study upon patients with congenital and infantile cataract for a period of 3 years (2009-2012). The study group included 36 children, and a number of 58 eyes.

In our study were included children of both gender with congenital or infantile cataract, aged between 2 months and 13 years, with primary implantation of intraocular lenses (IOL). From the study were excluded the cases of traumatic or complicated cataract, microcornea, microphthalmia, uveitis, aniridia, congenital glaucoma, persistent hiperplastic primary vitreous or retinal disease.

Ocular exam was performed before surgery at both eyes and included: visual acuity testing (at very young children we observed the ability of fixation or follow light; at the older one we used the Snellen chart); ocular refraction after cicloplegia, where it was possible (we used the PlusOptix System or the autorefractokeratometer (Topcan, Japan); keratometry - if possible; slit lamp examination in order to get information regarding the cornea, anterior chamber depth, iris, pupil and location and extension of the lens opacities; ocular motility; presence or absence of nystagmus or strabismus; tonometry - where it was possible (using the I-care tonometer, in order to rule out any association with glaucoma); measurement of corneal diameter; fundus examination, were it was possible.

In older and cooperative children, for the ocular biometry we used the Ocuscan biometer (Alcon, Forth Worth Tx). The axial length of the eye (AXL) was measured by A-scan ultrasonography contact method. The ultrasound velocities used were $1641 \mathrm{~m} / \mathrm{sec}$ for lens. In very young children we used B-scan biometry in order to measure the AXL of the eye. The formulas for the calculation of IOL dioptric power were the standard one: Hoffer Q, SRK-T or Holladay, concording with the AXL.

Correspondence to: Dr. Cristina Nicula, MD; PhD, Associate Professor, ClujNapoca University Ophthalmology Clinic, Cluj County Hospital - No. 3-5, Clinicilor Street, 400006, Cluj-Napoca, Romania, Tel: +40 722849 575; E-mail: niculacristina65@yahoo.com

Key words: congenital, infantile, cataract, ocular biometry

Received: August 04, 2016; Accepted: August 17, 2016; Published: August 22,2016 
The target refraction following pediatric cataract surgery is hyperopia, upon the age of the child: $+9 \mathrm{D}$ (where congenital cataract surgery is carried out at 4 to 8 weeks), $+6 \mathrm{D}$ (where the operation is done at 6 months), $+4 \mathrm{D}$ (where the operation is performed at 12 months), $+2 \mathrm{D}$ (where surgery is done at 2 years), $+1-+2 \mathrm{D}$ (where surgery is performed after 3 years of age), emetropie: where surgery is done after 11 years of age.

The measurement of dioptric power of IOL was made for in the bag implantation of the artificial lens. All the children received a hydrophobic acrylic IOL (Acrysof IQ - Alcon).

For statistical analysis we used the "Student t-test" and the "Pearson's correlation coefficient". The last coefficient assessed the relationship between the patient's age (in months) and ocular measurements (axial length and keratometry). The paired t-test was used to compare measurements from the unilateral eye with cataract and the healthy fellow eye. It was considered statistically significant when $\mathrm{p}<0.005$.

\section{Results}

All the patients were Caucasian, with ages between 4 and 216 months of age; four eyes from 58 eyes ( 36 children) were under 12 months of age and 8 eyes from 58 eyes ( 36 children) were between 12 and 36 months of age. The majority of cases ( 30 eyes from 15 children) were between 37 to 72 months of age. Only 16 eyes (from 36 children) were below 120 months. The majority of the children were male (26 eyes) (Figure 1).

More than half of the cases were males (26) where 10 cases were females (Figure 2). Regarding the unilaterality or bilaterality, we had 14 patients (14 eyes) with unilateral cataract and 22 patients (44 eyes) with bilateral cataract (Figure 3).

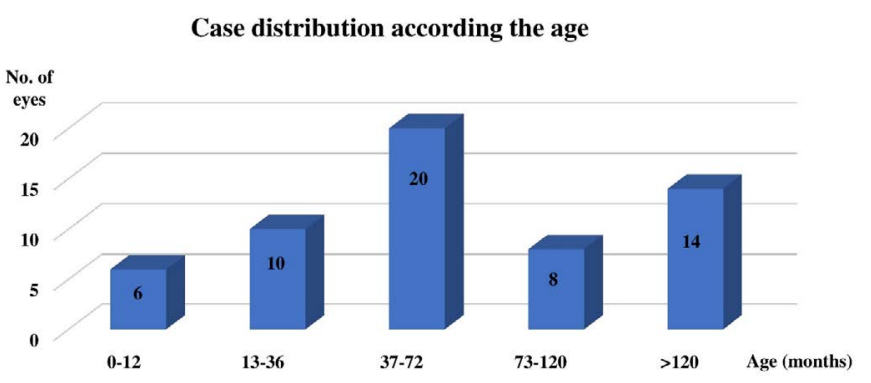

Figure 1. Cases repartition according to age (months).

\section{Case distribution according the gender}

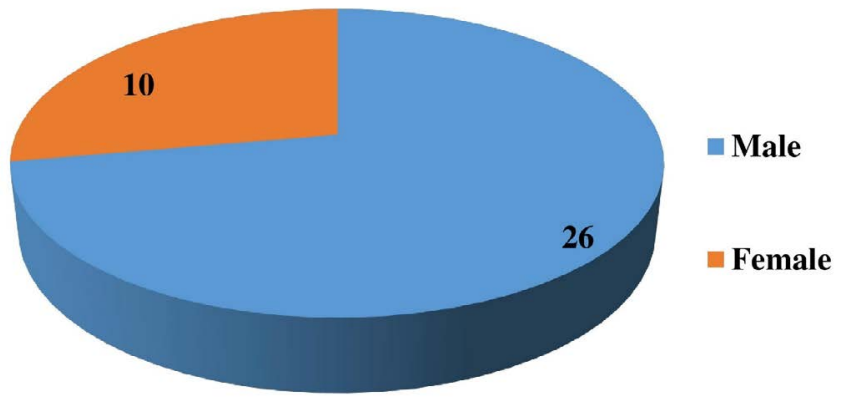

Figure 2. Cases repartition according to gender.
Regarding the type of the cataract we had in 40 eyes congenital cataract and the rest (18 eyes) were infantile cataracts (Figure 4 ).

The majority of the congenital cases (27 eyes) were of zonular opacification type, total cataract was present at 10 eyes, nuclear opacities at 17 eyes and posterior polar at 4 eyes (Figure 5).

At our patients, the measurement of AXL revealed an eye length between 20 and above $25 \mathrm{~mm}$. In 11 eyes we had AXL between 20 $21 \mathrm{~mm}$ and 15 eyes with AXL between 22-23 mm (Figure 6). The correlation between AXL and age showed a rapid increase in the first 12 months $(0.53 \mathrm{~mm} / \mathrm{month})$, a smaller increase between $100-150$ months $(0.1 \mathrm{~mm} / \mathrm{month})$ and stabilisation after 200 months. The data were statistically significant with $\mathrm{p}<0.05$ and $\mathrm{r}=0.41$ (medium correlation) (Figure 7).

The correlation between $\mathrm{Km}$ and age showed higher values of $\mathrm{Km}$

\section{Cases distribution by uni/bi-lateral cataract}

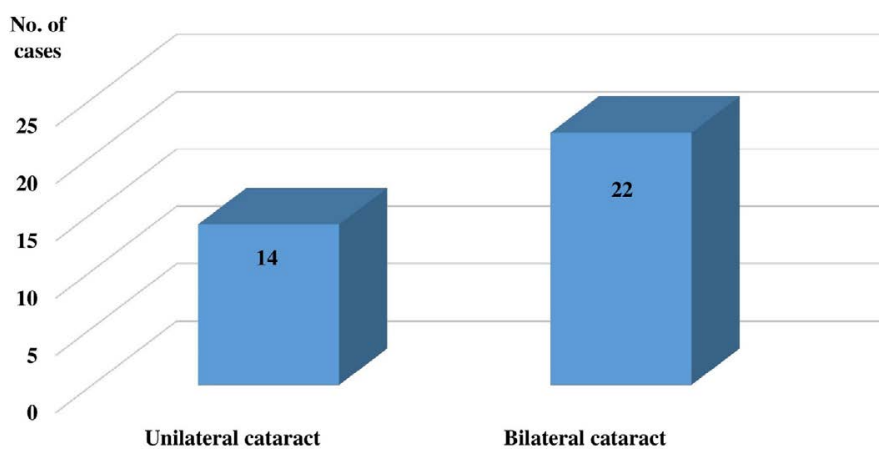

Figure 3. Cases repartition according touni/bi-lateral cataract.

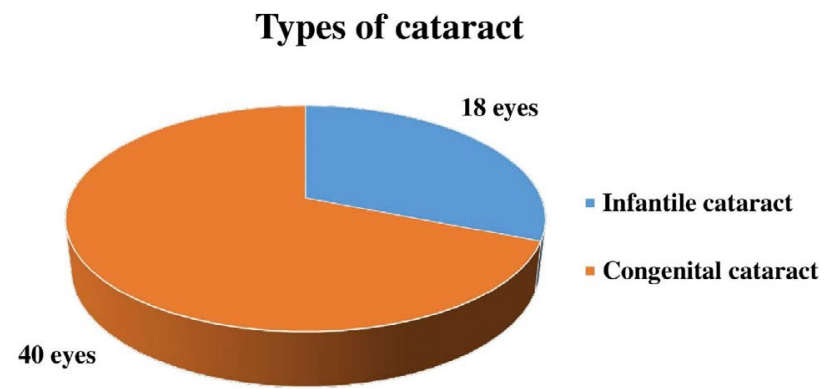

Figure 4. Cases repartition according to cataract type.

Types of congenital cataract

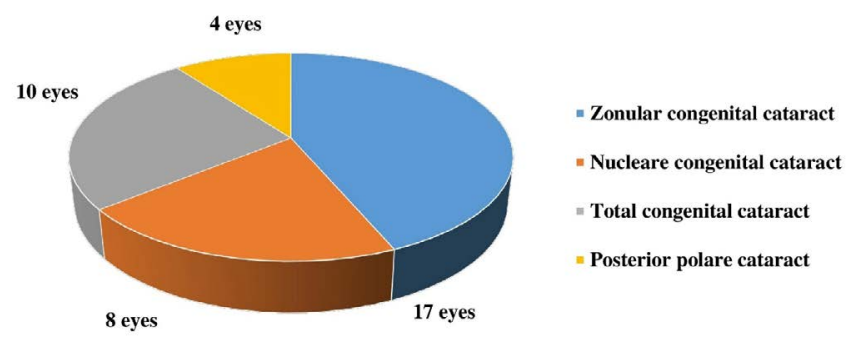

Figure 5. Cases repartition according to type of opacification 
at younger children, with a significant decrease in the first 12 months $(-0.5 \mathrm{D} /$ month $)$ and a smaller rate of decrease $(-0.1 \mathrm{D} /$ month $)$ after 24 months of age (Figure 8 ). The data were statistically significant, $\mathrm{p}<0.05$ and $\mathrm{r}=0.5$ (high correlation).

A comparison of ocular parameters in unilateral cataract with the fellow healthy eye showed that $\mathrm{Km}$ and AXL were greater in the cataractous eye comparing with the clear lens eye (Figure 9-10). The results were statistically significant with $\mathrm{p}$ value $<0.05$ for $\mathrm{Km}$ and AXL. Comparing AXL from unilateral cataract with one eye from bilateral cataract, we found that the AXL from unilateral cataract was significantly greater than the AXL from bilateral cataract ( $\mathrm{p}$ value $<$ 0.05) (Figure 3).

We didn't find any significant difference between the AXL from bilateral cataract (right eye, left eye) ( $p$ value $>0.05$ ) (Figure 11). Comparing the estimated target refraction and postoperative ocular refraction we had 3 eyes with a myopic shift of -4 iopters, 7 eyes with a myopic shift of -2 diopters even though the results were good ( $\mathrm{p}$ value $<0.05$ ) (Figure 12).
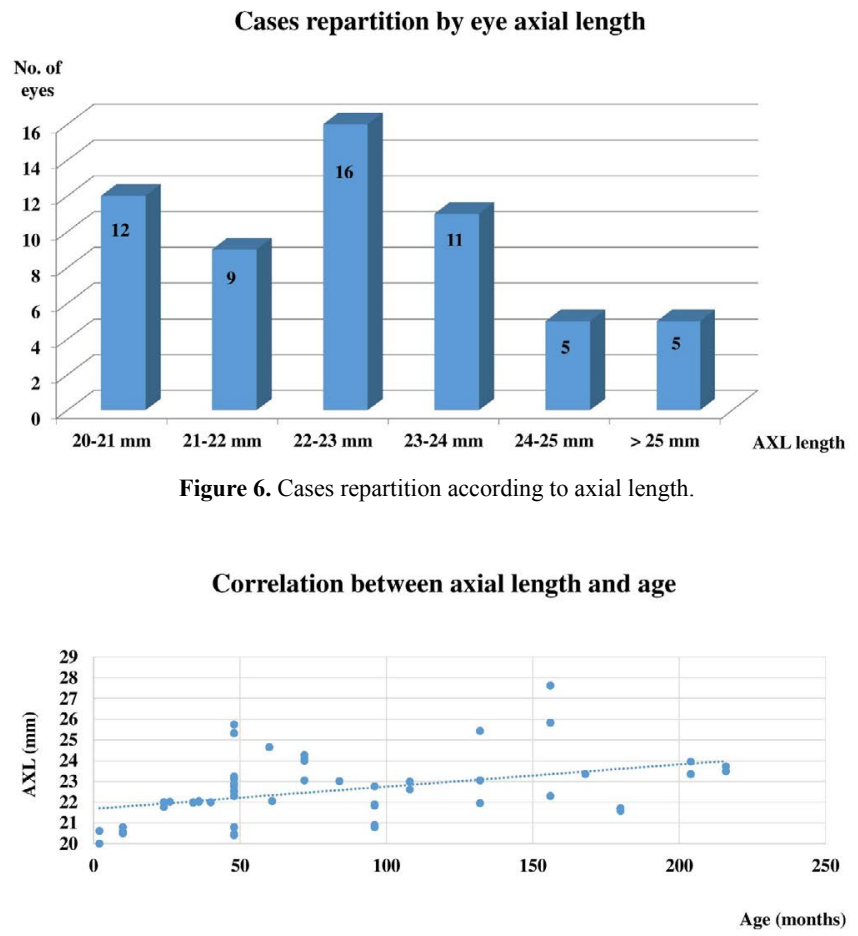

Figure 7. Correlation between axial length and age.

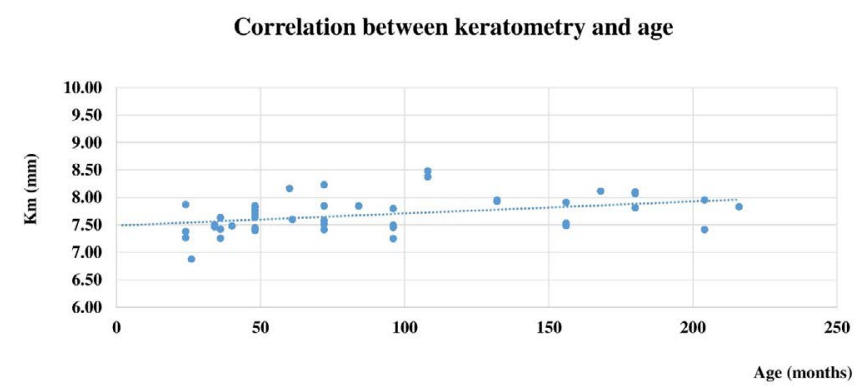

Figure 8. Correlation between keratometry and age.
Keratometry in unilateral cataract vs. keratometry in fellow eye

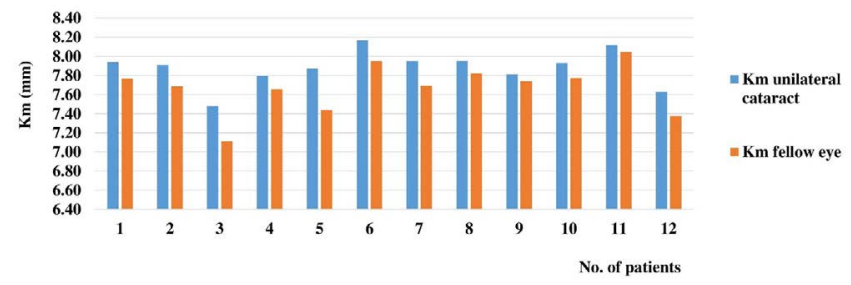

Figure 9. Keratometry in unilateral cataract vs. keratometry in fellow eye.

Axial length in unilateral cataract vs axial length in fellow eye

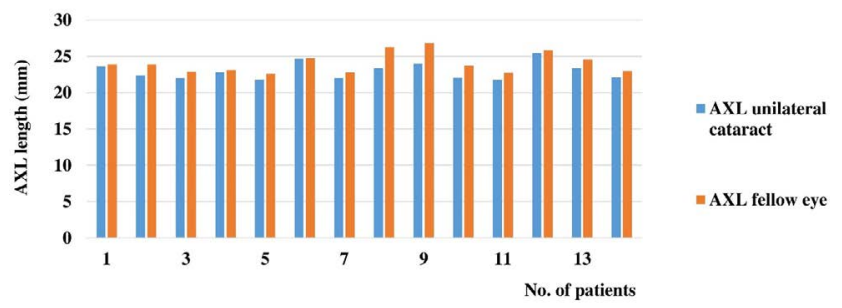

Figure 10. Axial length in unilateral cataract vs. axial length in fellow eye. Axial length in bilateral cataractat right eye vs.
axial length at bilateral cataract at left eye

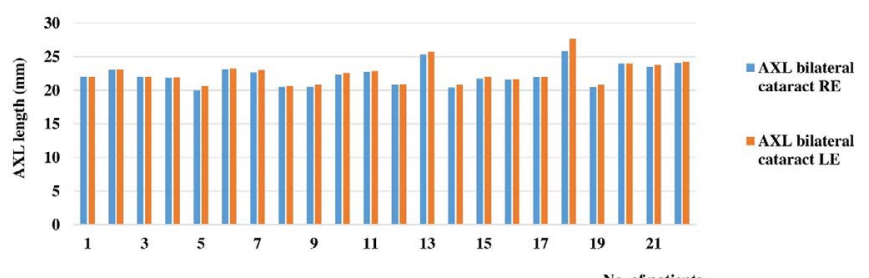

Figure 11. Axial length in bilateral cataract at right eye vs. axial length in bilateral cataract at left eye.

\section{Target refraction vs. postoperative refraction}

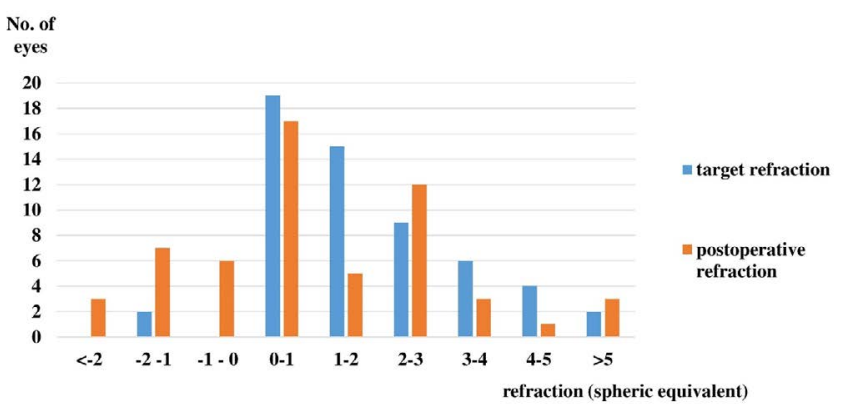

Figure 12. Target refraction vs. postoperative refraction.

\section{Discussions}

Ocular biometry in congenital/infantile cataract is very important for the visual outcomes but also very challenging because of the lack of cooperation. That's why to performing ocular biometry on children needs general anaesthesia.

The formulas used for biometry are those for adult people, 
concordant with the AXL of the eye. There are studies [5] which showed that Hoffer Q formula it's more predictive. Dahan et al. [6,7] showed that SRK-T formula was the best. Eibschitz Tsimhoni et al. [8] concluded that the error in IOL calculation is $0.8-1.3 \mathrm{D} / \mathrm{D}$ error in both children and adults. Mittelviefhans et al. [9] evaluated errors in keratometry due to lack of fixation in children who had keratometry under general anaesthesia. Their conclusion was that in order to improve the accuracy of the results multiple $\mathrm{K}$ readings should be taken. Hug et al. [10] concluded that the use of the aphakic refraction to calculate $\mathrm{AXL}$ and $\mathrm{K}$ value offers an alternative in paediatric patients when ultrasonic or patients sedation was not possible.

In our study, using the formulas concording with the AXL length we had 3 eyes (\%) with a myopic shift of $-4 \mathrm{D}$ and 7 eyes (\%) with a myopic shift of $-2 \mathrm{D}$. the results were statistically significant $(\mathrm{p}=0.005)$. We used for AXL $<22 \mathrm{~mm}$ the Hoffer $\mathrm{Q}$ formula, for AXL between 22$24 \mathrm{~mm}$ - Hoffer Q formula and for AXL > $24 \mathrm{~mm}$ - SRK-T formula.

Regarding the refractive target we established to be hyperopia, concording the age. Wilson et al. [5] concluded that most surgeons aim for moderate hyperopia ( $3 \mathrm{D}$ and $<7 \mathrm{D}$ ) in infants under 12 months and mild $(<3 \mathrm{D})$ to moderate hyperopia in infants at 12 months. Crouch et al. [11] recommended that the refractive goal for 2-4 years is $+2-+3 \mathrm{D}$, for $4-6$ years is $+1-+2 \mathrm{D}$ and for 10 years is $+1 \mathrm{D}$. Neely et al. [12] found that $23 \%$ of the pediatric patients had a refractive outcome more than $2 \mathrm{D}$ from the anticipated result and the variability outcomes were found especially in children younger than 2 years of age and an AXL $<19 \mathrm{~mm}$.

Khan et al. [13] in a retrospective study found that the ultrasonic AXL value and estimated AXL values had a difference of $0.05 \mathrm{~mm}$ and were not significantly different.

In our study the children had AXL lengths between 20 to $25 \mathrm{~mm}$. We concluded that the AXL length had a rapid increase in the first 12 months of age $(0.53 \mathrm{~mm} / \mathrm{month}) \mathrm{a}$ and a stabilisation after 200 months. Cappozzi et al. [4] obtained the same results.

Trivedi and Wilson [14] studied a large series of cataract patients whose age ranged between 1 month to 18 years. Their results are broadly consistent with our findings. They showed shorter AXL in girls than in boys.

Regarding both eyes, we found AXL significantly greater in eyes from unilateral versus bilateral cataract $(\mathrm{p}=0.00064)$. Trivedi and Wilson [14] showed the same results after 60 months of age. We didn't find any difference in AXL length between right eye and left eye in bilateral cases $(\mathrm{p}=0.7)$. Also UD Shrestha et al. [15] in their study showed that the mean value of axial length in different age group is nearly equal in operated and fellow eye, with no significant modification (p.0.05).

Regarding unilateral pediatric cataract, we found that AXL length in the cataractous eye was shorter than in the noncataractous eye $(\mathrm{p}=0.03)$. Kugelberg et al. [16] showed the same results. Shrestha et al. [15] showed that in unilateral congenital cataract the AXL length in the affected eye is shorter than the healthy eye and the lens is thinner.

Cappozzy et al. [4] didn't find any difference between AXL in unilateral cataract with the fellow eye. Maybe the different results in our study can be explained that we used the same ultrasound velocity for both eyes. In the cataractous eye the ultrasound propagation is faster and may lead to an underestimation of AXL in the affected eye.

Regarding the $\mathrm{Km}$ value and age, our study showed a significant decrease of $\mathrm{Km}$ in the first 12 months $(0.5 \mathrm{D} /$ month). We also found a small rate of decrease after 24 months of age $(-0.1 \mathrm{D} /$ month). Trivedi and Wilson [14] showed in their study that Km stabilises by 6 months of age, but they showed steeper corneas and shorter AXL in girls than in boys. Cappozzi et al. [4] found that modification in $\mathrm{Km}$ continue in the second semester and also in the second year of life, although at a lower rate. The same results were shown by Ehlers et al. [17].

In our study we had a myopic shift of $4 \mathrm{D}$ in 3 eyes and a myopic shift of $2 \mathrm{D}$ in 7 eyes. Crouch et al. [11] reported a myopic shift of 3.66 $\mathrm{D}$ in children operated in 3-4 years of age, 2.03 D in children operated in 7-8 years and $1.88 \mathrm{D}$ ion children operated on 9 years. Some studies [18] showed that the implantation of a PC-IOL may retard the axial elongation of the eye comparing with the aphakic eyes. Plager [19], Wilson [20] and Dahan [6] reported almost the same results regarding the myopic shift. The conclusion was that children aged 2 years and under at the time of surgery had a significantly greater predicted myopic shift and a greater variance in the predictive refractive change than those older than 2 years at the time of surgery.

\section{Conclusions}

In conclusion our study shows that the AXL length increases during childhood, especially in the first 2 years and the corneal curvature decreases from birth to different ages, parameters which induce the target refraction.

\section{References}

1. Morselei S, Belenei R, Pearls and tricks in congenital cataract surgery. In Lee SA et al., Pediatric Ophthalmology. Ed. Jaypee Brothers Medical Publisher LTD, St. Louis; 2009:34.

2. Lloyd IC1, Ashworth J, Biswas S, Abadi RV (2007) Advances in the management of congenital and infantile cataract. Eye (Lond) 21: 1301-1309. [Crossref]

3. Ashworth J, Lloyd C (2007) How to achive optimal refractive outcomes. Ophthalmology Times Europe: 8. URL: http://www.oteurope.com/ophthalmologytimes europe/article/ article.

4. Capozzi P, Morini C, Piga S, Cuttini M, Vadalà P (2008) Corneal curvature and axial length values in children with congenital/infantile cataract in the first 42 months of life. Invest Ophthalmol Vis Sci 49: 4774-4778. [Crossref]

5. Wilson ME, Bartholomew LR, Trivedi RH (2003) Pediatric cataract surgery and intraocular lens implantation: practice styles and preferences of the 2001 ASCRS and AAPOS memberships. J Cataract Refract Surg 29: 1811-1820. [Crossref]

6. Nihalani BR, VanderVeen DK (2010) Comparison of intraocular lens power calculation formulae in pediatric eyes. Ophthalmology 117: 1493-1499. [Crossref]

7. Kekunnaya R, Gupta A, Sachdeva V, Rao HL, Vaddavalli PK, et al. (2012) Accuracy of intraocular lens power calculation formulae in children less than two years. $\mathrm{Am} \mathrm{J}$ Ophthalmol 154: 13-19. [Crossref]

8. Vanderveen DK, Trivedi RH, Nizam A, Lynn MJ, Lambert SR (2013) Infant Aphakia Treatment Study Group. Predictability of intraocular lens power calculation formulae in infantile eyes with unilateral congenital cataract: results from the Infant Aphakia Treatment Study. Am J Ophthalmol 156: 1252-1260.e2.

9. Dahan E, Drusedau MU (1997) Choice of lens and dioptric power in pediatric pseudophakia. J Cataract Refract Surg 23 Suppl 1: 618-623. [Crossref]

10. Dahan E (2000) Intraocular lens implantation in children. Curr Opin Ophthalmol 11 51-55. [Crossref]

11. Eibschitz-Tsimhoni M, Tsimhoni O, Archer SM, Del Monte MA (2008) Effect of axial length and keratometry measurement error on intraocular lens implant power prediction formulas in pediatric patients. JAAPOS 12: 173-176. [Crossref]

12. Mittelviefhaus H, Gentner C (2000) Errors in keratometry for intraocular lens implantation in infants. Ophthalmologe 97: 186-188. [Crossref]

13. Abdel-Hafez G, Trivedi RH, Wilson ME, Bandyopadhyay D (2011) Comparison of aphakic refraction formulas for secondary in-the-bag intraocular lens power estimation in children. JAAPOS 15: 432-434. [Crossref]

14. Crouch ER, Crouch ER Jr, Pressman SH (2002) Prospective analysis of pediatric 
pseudophakia: myopic shift and postoperative outcomes. J AAPOS 6: 277-282. [Crossref]

15. Neely DE, Plager DA, Borger SM, Golub RL (2005) Accuracy of intraocular lens calculations in infants and children undergoing cataract surgery. J AAPOS 9: 160-165. [Crossref]

16. Hoevenaars NE, Polling JR, Wolfs RC (2011) Prediction error and myopic shift after intraocular lens implantation in paediatric cataract patients. Br J Ophthalmol 95: 10821085. [Crossref]

17. Khan AO (2007) Emmetropisation after lensectomy and anterior vitrectomy for persistent hyperplastic primary vitreous cataract. Eye Contact Lenses 33: 199-200. [Crossref]

18. Trivedi RH, Wilson ME (2007) Biometry data from caucasian and african-american cataractous pediatric eyes. Invest Ophthalmol Vis Sci 48: 4671-4678. [Crossref]

19. UD Shrestha, MK Shrestha (2011) Axial length in eyes with bilateral cataract at tertiary eye care center in Nepal: A preliminary report. Nepal Med Coll J 13: 62-63. [Crossref]

20. Kugelberg U, Zetterström C, Syrén-Nordqvist S (1996) Ocular axial length in children with unilateral congenital cataract. Acta Ophthalmol Scand 74: 220-223. [Crossref]

21. Trivedi RH, Wilson ME (2011) Prediction error after pediatric cataract surgery with intraocular lens implantation: contact versus immersion A-scan biometry. $J$ Cataract Refract Surg 37: 501-505. [Crossref]

22. Ehlers N, Sorensen T, Bramsen T, Poulsen EH (1976) Central corneal thickness in newborns and children. Acta Ophthalmol (Copenh) 54: 285-290. [Crossref]

23. Griener ED, Dahan E, Lambert SR (1999) Effect of age at time of cataract surgery on subsequent axial length growth in infant eyes. J Cataract Refract Surg 25: 1209-1213. [Crossref]

24. Plager DA, Kipfer H, Sprunger DT, Sondhi N, Neely DE (2002) Refractive change in pediatric pseudophakia: 6-year follow-up. J Cataract Refract Surg 28: 810-815. [Crossref]

25. Wilson ME, Peterseim MW, Englert JA, Lall-Trail JK, Elliott LA (2001) Pseudophakia and polypseudophakia in the first year of life. J AAPOS 5: 238-245. [Crossref]

26. VanderVeen DK, Nizam A, Lynn MJ, Bothun ED, McClatchey SK, et al. (2012) Predictability of intraocular lens calculation and early refractive status: the Infant Aphakia Treatment Study. Arch Ophthalmol 130: 293-299.

Copyright: (C2016 Nicula C. This is an open-access article distributed under the terms of the Creative Commons Attribution License, which permits unrestricted use, distribution, and reproduction in any medium, provided the original author and source are credited. 